\title{
Robotic distal subtotal gastrectomy with D2 lymphadenectomy for advanced gastric cancer: A case report and technical description
}

\author{
Francesco Giovanardi1, ${ }^{*}$ \\ 1 Department of General Surgery and Organ Transplantation "Paride Stefanini”, Sapienza University of Rome, Rome, Italy.
}

\section{To Cite}

Giovanardi F. Robotic Distal Subtotal Gastrectomy with D2 Lymphadenectomy for Advanced Gastric Cancer: A Case Report and Technical Description. J Gastric Surg 2020; 2(1): 18-21

\section{Pubblication history}

Received: February 4, 2020

Revised: February 28, 2020

Accepted: March 7, 2020

Article in press: March 8, 2020

Published online: March 15, 2020

\section{*Correspondence to}

Dr. Francesco Giovanardi

Department of General Surgery and Organ

Transplantation "Paride Stefanini",

Sapienza University of Rome,

Rome, Italy.

giovanardi89@gmail.com

Telephone: +393202137231

\begin{abstract}
Robotic systems have revolutionized the way we perform minimally invasive surgery and has facilitated the evolution of traditional laparoscopic gastric surgery. Surgeons have several advantages that can overcome some of the well-known limits of laparoscopy: three-dimensional vision, articulated instruments, the absence of tremors. These can give greater dexterity and precision in dissection and suturing movements that are key elements when performing complex and gentle reconstruction to restore digestive continuity.

The present case shows the technical details and tips and tricks of a robotic surgical approach for a subtotal gastrectomy.

Key words:

robotic surgery, gastric cancer, subtotal gastrectomy, minimally invasive surgery.
\end{abstract}




\section{Introduction:}

The robotic approach for technically demanding complex abdominal cancer operations, such as radical surgery for advanced gastric cancer, has been standardized to facilitate minimally invasive surgery[1-4]. Robotic subtotal gastrectomy with D2 lymphadenectomy using the DaVinci Xi Surgical System is described in the present case (St. Mary's Hospital of Terni).

\section{Case presentation:}

A 52-year-old woman with biopsy proven signet ring cell adenocarcinoma of the stomach was clinically staged cT3N1M0 after endoscopic ultrasound and a CT scan. She was found to have a prepyloric mass invading the subserosa and two pathological appearing lymph nodes in station \#6 without evidence of metastatic disease. After discussion of treatment options including upfront surgery followed by adjuvant treatment versus neoadjuvant treatment followed by surgery, the patient underwent a robotic distal subtotal gastrectomy with D2 lymphadenectomy.

\section{Procedure details:}

The procedure was performed with the patient in the supine position with arms tucked to the patient sides. Four $8 \mathrm{~mm}$ robotic trocars and one $15 \mathrm{~mm}$ assist trocar were placed.

The daVinci Xi Surgical System's bedside chart was brought in from the patient's right side and docked. The instruments were inserted into the robotic arms (Arm 1. cadiere forceps; Arm 2: harmonic ultrasonic shears / cautery hook; Arm 3: Camera; Arm 4: Maryland bipolar). The primary surgeon moved to the surgeon console and the procedure began with the division of gastrocolic ligament using the harmonic scalpel from right to left and proximally to identify and divide, between hem-o-lock, the left gastroepiploic artery and retrieve lymph node station $\# 4 \mathrm{sb}$. The level of the proximal resection is also identified. Then, the greater curvature was cleared of \#4d, including the lymph nodes along the second branch and distal part of the right gastroepiploic artery (RGEA).

The division of the gastrocolic ligament continued distally. To do that, the posterior surface of the stomach should be well lifted up, exposing the pancreatico-duodenal area. The superior right colic vein was identified in order to find the gastrocolic trunk of Henle (Figure 1).

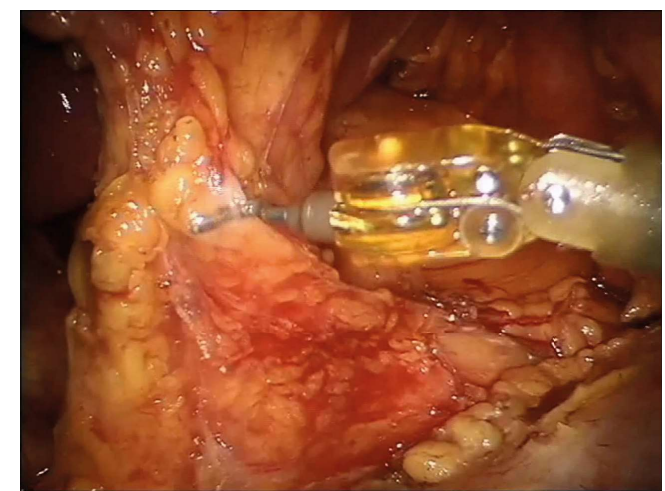

Figure 1: Origin of the right gastroepiploic vessels and infrapyloric lymphnodes (\#6).

The latter was dissected, and the right gastroepiploic vein was sectioned at its origin.

The base of the right gastroepiploic vein was identified and isolated, as it entered the superior mesenteric vein and divided between hem-o-lock.

The posterior gastric wall was completely mobilized from the anterior surface of the pancreas by sectioning all adhesions with the pancreatic capsule.

The right gastroepiploic artery was identified at its origin from the gastroduodenal artery and divided as well. This allowed for the en-block retrieval of \#6, the soft tissue along the proximal part of the RGEA and on the anterior surface of the head of the pancreas above the anterior superior pancreaticoduodenal vein.

Here, the pylorus and the first portion of the duodenum need to be completely released and particularly the entire course of the gastroduodenal artery behind the duodenum, marking the distal visceral resection.

The duodenocolic ligament was divided and the infraduodenal and supradudonal area were cleared allowing for the division of the duodenum.

The assistant introduced, through the assistant port, an articulated linear mechanical stapler with a visceral cartridge, placing and firing it $1 \mathrm{~cm}$ downstream from the pylorus.

This step needs to be well coordinated by the robotic surgeon in order to obtain the correct angle of section. However, the primary surgeon can decide to perform that by himself with a robotic stapler.

Now, the extra-gastric lymphadenectomy begins along the proper hepatic artery.

The soft tissue anterior to the artery was cleared identifying the base of the right gastric artery and allowing for retrieval of \#5.

The proper hepatic artery was identified and isolated up the hepatic pedicle, in the context of the hepatoduodenal ligament. The soft tissue anterior to and medial to the portal vein along the proper hepatic artery was cleared, retrieving \#12a.

The peri-hepatic major vessels were completely released of their perivascular tissue.

Now, the dissection can continue at the level of the upper edge of the pancreas (Figure 2).

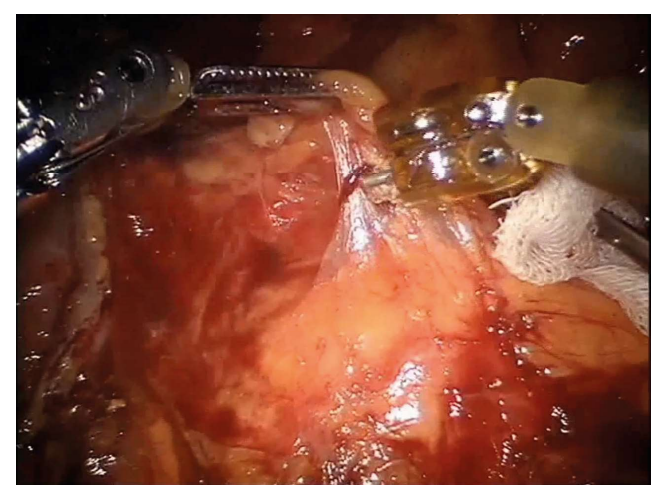

Figure 2: Dissection along the common hepatic artery (limphnode station \#8).

The common hepatic artery was stripped of the lymphatic tissue from the origin of the gastro-duodenal artery to the celiac axis, \#8.

The left gastric vein was reached, in this case anterior to the celiac axis (Figure 3).

The Maryland bipolar helped in this case to isolate the left gastric vein from the other vessels. Then, it was divided, offering access to \#9 that was cleared lateral to the celiac 
axis.

At this point, the base of the left gastric artery was identified, clipped with hem-o-lock and divided, clearing \#7.

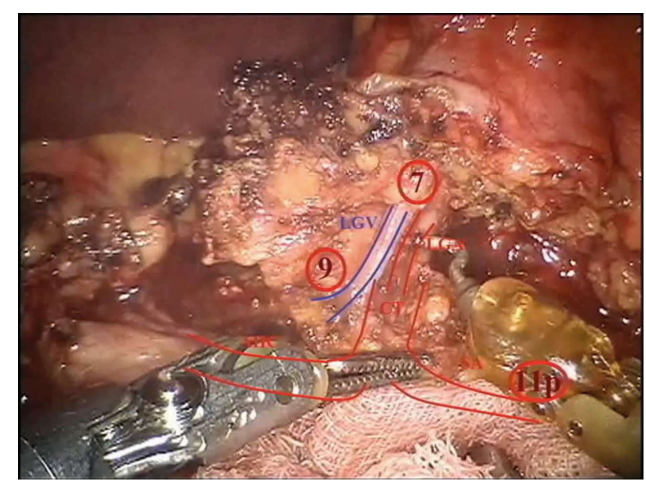

Figure 3: Coeliac trunk (CT) and its branches (common hepatic artery CHA, left gastric artery LGA, splenic artery SA). The left gastric vein (LGV) is anterior to the LGA. View of limphnode stations \#9 and \#7.

The course of the splenic artery was identified and the dissection prolonged to include the proximal splenic artery lymph nodes from its origin to halfway between its origin and the pancreatic tail end (Figure 4).

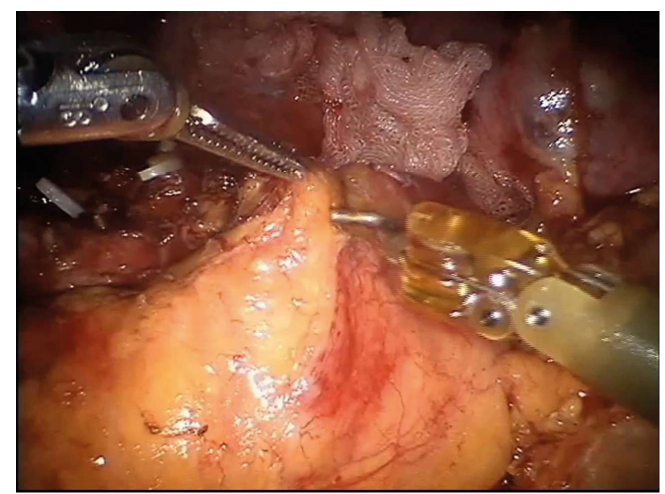

Figure 4: Dissection along the proximal part of the splenic artery.

At this point, the D2 lymphadenectomy was completed by removing the soft tissue along the esophageal crus, clearing the right paracardial nodes, \#1, as well as that along the lesser curvature including all nodes along the lower branch of the left gastric artery up to the right gastric artery, \#3.

All vessels should be well identified after a D2 lymphadenectomy, particularly the common hepatic artery which surrounds the upper edge of the pancreas and the right and left gastric artery, divided at their origins from the proper hepatic artery and the celiac axis, respectively.

The proximal stomach was resected with the linear stapler introduced by the assistant. A loop of small bowel was identified and an intra-corporeal side-to-side anastomosis was created (Figure 5).

The umbilical incision was extended to about $4 \mathrm{~cm}$ to remove the specimen.

Patient followed an enhanced recovery after surgery protocol and was discharged on fifth postoperative day without complications.

Final pathology revealed a pT3N2 poorly differentiated adenocarcinoma with 76 nodes examined.

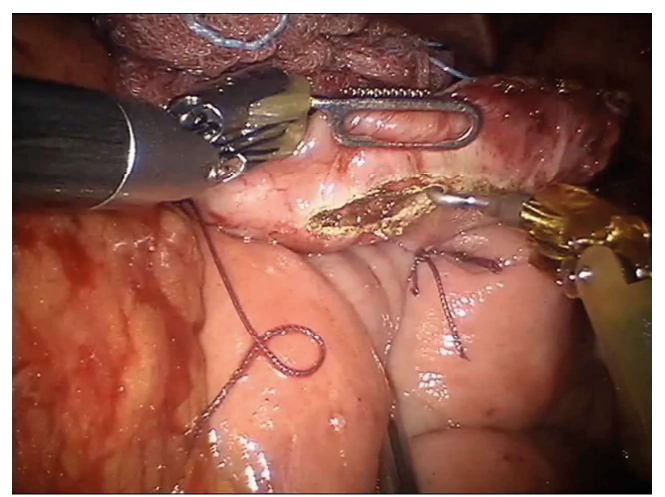

Figure 5: Reconstruction phase: Billroth II gastrojejunostomy.

\section{Conclusion:}

The robotic system can enhance a minimally invasive oncological dissection, allowing a complete removal of the soft tissue around major vessels, considered the most challenging step for a complete D2 gastrectomy.

\section{Acknowlwdgements \\ Not applicable}

\section{Contributors}

FG conceptualized and designed the study, acquired and analysed data, drafted and revised the final manuscript.

\section{Funding}

None.

\section{Competing interests}

No benefits in any form have been received or will be received from a commercial party related directly or indirectly to the subject of this article.

\section{Availability of data and materials}

Further information are available from the corresponding author on reasonable request.

\section{Ethics approval}

Informed consent was obtained from the patients for being included in the study.

\section{Provenance and peer review}

Not commissioned; externally peer reviewed.

\section{Open access}

This is an Open Access article distributed in accordance with the Creative Commons Attribution Non-Commercial (CC BY-NC 4.0) license, which permits others to distribute, remix, adapt, build upon this work noncommercially, and license their derivative works on different terms, provided the original work is properly cited and the use is noncommercial. See: http:/ / creativecommons.org/licenses/ by-nc/4.0/

\section{References}

[1] Desiderio J, Jiang ZW, Nguyen NT, Zhang S, Reim D, Alimoglu O, et al. Robotic, laparoscopic and open surgery for gastric cancer compared on surgical, clinical and oncological outcomes: a multi-institutional chart review. A study protocol of the International study group on Minimally Invasive surgery for GASTRIc Cancer-IMIGASTRIC. BMJ Open. 2015;5:e08198

[2] Desiderio J, Stewart CL, Sun V, Melstrom L, Warner S, Lee B, et al. Enhanced Recovery after Surgery for Gastric Cancer Patients Improves Clinical Outcomes at a US Cancer Center. J Gastric Cancer. 2018;18:230- 
41.

[3] Parisi A, Nguyen NT, Reim D, Zhang S, Jiang ZW, Brower ST, et

al. Current status of minimally invasive surgery for gastric cancer: A literature review to highlight studies limits. Int J Surg. 2015;17:34-40.

[4] Parisi A, Reim D, Borghi F, Nguyen NT, Qi F, Coratti A, et al. Minimally

invasive surgery for gastric cancer: A comparison between robotic,

laparoscopic and open surgery. World J Gastroenterol. 2017;23:2376-84. 